\title{
Candidemia Profile and Antifungal Drugs Susceptibility Pattern in Neonatal Intensive Care Unit Patients in a Tertiary Care Teaching Institute in Maharashtra
}

\author{
Vanita Ashok Kulkarni ${ }^{1}$, Arati Ankuhrao Bhadade ${ }^{2 *}$ and Shilpa Dayanand Putta ${ }^{1}$ \\ 'Department of Microbiology, Rajashri Chhatrapati Shahu Maharaj \& Government Medical College, Kolhapur, India \\ ${ }^{2}$ Department of Microbiology, Government Medical College, Nagpur. India
}

\begin{abstract}
Background: Invasive candidiasis is one of the most commonly encountered problems in neonatal intensive care unit (NICU) leading to substantial morbidity and mortality. Recent studies show an increase in antifungal resistance of candida species. Therefore, species identification with antifungal susceptibility pattern of Candida isolates is very important and helps in the selection of appropriate antifungal agents to prevent the emergence of drug resistance and successful treatment.

Methods: Seven thirty four blood culture samples from 244 patients of NICU were tested. Yeast isolates from these blood cultures were identified by conventional methods. Anti-fungal susceptibility testing was done according to CLSI M44A guidelines for Fluconazole, Voriconazole, ketoconazole, Amphotericin-B and Itraconazole.

Result: From 118 isolates c. albicans were $37.28 \%$ and remaining $62.72 \%$ were non-albicans candida species including c.glabrata(28.81\%), c.parapsilosis(14.4\%), c.tropicalis(12.71\%) and other(6.77\%). From all isolates of candida species, Fluconazole, Amphotericin B and ketoconazole were sensitive in $61.0 \%, 86.4 \%$ and $99.1 \%$ patients respectively. Voriconazole and Itraconazole were $100 \%$ sensitive.

Conclusion: Although previously Candida albicans accounted for majority cases of candidiasis, recently NAC species have been increasingly reported in neonatal intensive care units. Amphotericin B should be reserved for life threatening conditions and extended spectrum azole drugs like Voriconazole, Itraconazole and ketoconazole can be good alternatives for treating these infections.
\end{abstract}

\section{Keywords: Antifungal Drug Resistance, AFST , Candidemia , NICU}

\section{Introduction}

Invasive candidiasis is one of the most commonly encountered problems in neonatal intensive care units (NICU). Number of factors including the use of indwelling devices, broad spectrum antibiotics, low birth weight (LBW), prematurity, total parenteral nutrition (TPN), gastrointestinal surgery, artificial ventilation, and/or history of fungal colonization contribute to the risk. ${ }^{[1]}$ Preterm, very low birth weight (VLBW): $\leq 1,500 \mathrm{~g}$; extremely low birth weight (ELBW): $\leq 1,000 \mathrm{~g}$; and critically ill infants are at highest risk of invasive Candida infections. ${ }^{[2]}$ Candida spp. can also spread through vertical transmission from maternal flora or via horizontal transmission from hands of healthcare workers (HCW) ${ }^{[3]}$

Candidemia currently rank as the fourth most common cause of nosocomial bloodstream infections. ${ }^{[4]}$ The associated crude mortality is high $(38 \%-75 \%)$ despite appropriate treatment with antifungal agents. ${ }^{[5]}$ It is the third most common cause of late onset sepsis in NICU patients and accounts for $9-13 \%$ of blood stream infections (BSI) in neonates. ${ }^{[6]}$ Invasive candidiasis is associated with substantial morbidity and mortality and is difficult to diagnose due to lack of specific signs and symptoms. ${ }^{7]}$ The relationship between prognosis and early initiation of the adequate antifungal therapy is well established. ${ }^{[7]}$

The last two decades have seen a significant rise in the infections caused by Candida species in various centers in India. Although previously Candida albicans account for majority cases of candidiasis, recently non-albicans Candida (NAC) species have been increasing reported. ${ }^{[4]}$ Less than $50 \%$ of all candidal blood isolates are C. albicans whereas Candida glabrata, Candida parapsilosis, Candida tropicalis, and other NAC account for rest of the candidial blood stream infection. ${ }^{[8]}$ There is growing evidence suggesting a role of increasing use of azole agents in this epidemiological shift. Recent studies show an increase in antifungal resistance of candida species. Several of these NAC species like C. krusei and C. glabrata exhibit intrinsic resistance to traditional triazoles like fluconazole (FLK) and may also demonstrate cross resistance to newer triazoles. ${ }^{[9]}$ Therefore, species identification with antifungal susceptibility pattern of Candida isolates is very important and helps in the selection of appropriate antifungal agents, successful treatment, in antifungal prophylaxis in the 
immunocompromised host and to prevent the emergence of drug resistance. ${ }^{[10]}$

There has been attempts and subsequent successes in developing reliable methods for antifungal susceptibitity testing of candida species. Although there has been standardized guidelines for microdilution methods (M27-A2), it has not been routinely used in majority of laboratories because of its complexcity and cost. ${ }^{[1]}$ Recently CLSI has given guidelines for antifungal susceptibitity testing of yeast by disk diffusion method(M44-A). [12] This method is more practically feasible for majority of laboratories. In particular, testing Candida species against azole antifungal agents has provided valuable information for treatment of patients with invasive yeast infections.

Clinicians now depend on identification of Candida to the species level in order to optimize the selection of antifungal agents and to allow them to provide the best possible patient care. Hence this study is done to evaluate candidemia infections in ICU so that the early detection of candidemia and antifungal susceptibility testing will give better prognosis and optimize the selection of antifungal agents in NICU patients.

\section{Materials and Methods}

The present study was carried out in department of Microbiology, tertiary care teaching hospital, western Maharashthra for period of 1 year from January to December 2015 after obtaining permission from institutional ethics committee. 734 blood culture samples from 244 patients of NICU were tested. Neonates in NICU suspected of fungal sepsis were included in this study. Patient already on antifungal drugs were excluded from this study.

Testing of Samples: Fungal blood culture bottles and short proforma were provided in NICU. Three blood cultures were taken from each patient after a gap of 48 hours. First blood culture was taken on suspicion of sepsis. Subcultures were done on Sabouraud's dextrose agar (SDA) with chloramphenicol, gentamicin without cycloheximide on day 1 , day $3 \&$ day 7 and incubated at $25^{\circ} \mathrm{C}$ and $37^{\circ} \mathrm{C}$ for one month. Readings were taken daily for 1 week \& then every alternate day. Yeast isolates from these blood cultures were identified by conventional methods like germ tube test, urease test and Dalmau's technique. ${ }^{[13]}$

Susceptibility Test Method ${ }^{[12]}: 24$ hour subcultures of these candida species were used for anti-fungal susceptibility testing (AFST) of Fluconazole $(25 \mu \mathrm{g})$, Voriconazole(1 $\mu \mathrm{g})$, ketoconazole $(10 \mu \mathrm{g})$, Amphotericin-B(100 units) and Itraconazole disks $(10 \mu \mathrm{g})$. All dehydrated culture media and antifungal discs were procured from Hi-Media. MuellerHinton agar(MHA) supplemented with $2 \%$ glucose (to support growth) and $0.5 \mu \mathrm{g}$ of methylene blue (improves zone edge definition) per $\mathrm{ml}$ was used for AFST. The inoculum was adjusted to match a $0.5 \mathrm{McFarland}$ density standard. After inoculation plates were incubated for 18 to $24 \mathrm{~h}$ at 35 to $37^{\circ} \mathrm{C}$. The plate reading was done according to CLSI M44A guidelines. Results were analysed by statistical methods. Candida albicans ATCC $90028^{[12]}$ was used as control.

\section{Result}

Two fourty four patients of NICU suspected of fungal infection were enrolled in this study. Out of total 244 neonates $146(59.8 \%)$ were males and 98 (40.2\%) were females. The male to female ratio was 1.5: 1 .

Seven thirty four blood culture samples of these 244 patients were taken. Out of these 734 blood culture samples 118 were culture positive where candida species were isolated.

Table 1 shows that from 118 isolates $c$. albicans were 37.28 $\%(44 / 118)$ and remaining $62.72 \%(74 / 118)$ were nonalbicans candida (NAC). From NAC 28.81\% (34/118) were c. glabrata, $14.4 \%(17 / 118)$ were c. parapsilosis, $12.71 \%$ $(15 / 118)$ c. tropicalis were c. parapsilosis and $6.77 \%$ (8/118) were other candida species (c. guilliermondii, $c$. krusei and c. kefyr). Table 1 shows that C.albicans was the most common isolated species and c. glabrata was the second most isoiated species.

Table 2 and Image 1 shows antifungal drug Susceptibility pattern of various Candida species. From all isolates of candida, fluconazole was resistant in $29.7 \%$, Intermediate in $9.3 \%$ and sensitive in $61.0 \%$ patients. Amphotericin B was resistant in $10.1 \%$, Intermediate in $3.4 \%$ and sensitive in $86.4 \%$ patients. Twelve isolates (5 glabrata, $4 C$. albicans, 2 C. tropicalis a nd 1 c. paropsilosis) were found resistant to amphotericin $\mathrm{B}$. ketoconazole was sensitive in $99.1 \%$ patients. Voriconazole and Itraconazole were 100\% sensitive. Overall, Voriconazole and Itraconazole were the most active agent in vitro and ketoconazole was next to them with only one isolate exhibiting resistance to it.

Table 1: Different species of Candida isolated from the culture positive cases.

\begin{tabular}{|c|c|c|}
\hline Species & no. of isolates & percentage (\%) \\
\hline c.albicans & 44 & 28.28 \\
\hline c.glabrata & 34 & 14.4 \\
\hline C.paropsilosis & 17 & 28.81 \\
\hline
\end{tabular}

Annals of Pathology and Laboratory Medicine, Vol. 04, No. 01, January - February, 2017 


\begin{tabular}{|c|c|c|}
\hline Species & no. of isolates & percentage (\%) \\
\hline c.tropicalis & 15 & 6.71 \\
\hline Other & 8 & 12.71 \\
\hline Total & 118 & 100 \\
\hline
\end{tabular}

Table 2 : Antifungal Susceptibility pattern of various Candida species.

\begin{tabular}{|c|c|c|c|c|c|c|c|c|c|c|c|c|c|c|c|}
\hline \multirow{2}{*}{ species (no.tested) } & \multicolumn{3}{|c|}{ Fluconazole } & \multicolumn{3}{|c|}{ Amphotericin B } & \multicolumn{3}{|c|}{ Voriconazole } & \multicolumn{3}{|c|}{ Itraconazole } & \multicolumn{3}{|c|}{ ketoconazole } \\
\hline & S & I & $\mathbf{R}$ & S & I & $\mathbf{R}$ & S & I & $\mathbf{R}$ & S & I & $\mathbf{R}$ & $\mathbf{S}$ & I & $\mathbf{R}$ \\
\hline c.albicans (44) & 26 & 6 & 12 & 39 & 1 & 4 & 43 & 0 & 0 & 44 & 0 & 0 & 43 & 0 & 1 \\
\hline c.tropicalis (17) & 10 & 1 & 6 & 15 & 0 & 2 & 17 & 0 & 0 & 17 & 0 & 0 & 17 & 0 & 0 \\
\hline c.paropsilosis (15) & 13 & 1 & 1 & 14 & 0 & 1 & 15 & 0 & 0 & 15 & 0 & 0 & 15 & 0 & 0 \\
\hline c.glabrata (34) & 17 & 3 & 14 & 26 & 3 & 5 & 33 & 0 & 0 & 34 & 0 & 0 & 34 & 0 & 0 \\
\hline other (8) & 6 & 0 & 2 & 8 & 0 & 0 & 8 & 0 & 0 & 8 & 0 & 0 & 8 & 0 & 0 \\
\hline Total (118) & 72 & 11 & 35 & 102 & 4 & 12 & 116 & 0 & 0 & 118 & 0 & 0 & 117 & 0 & 1 \\
\hline percentage (\%) & 61.0 & 9.3 & 29.7 & 86.4 & 3.4 & 10.1 & 100 & 0 & 0 & 100 & 0 & 0 & 99.1 & 0 & 0.9 \\
\hline
\end{tabular}

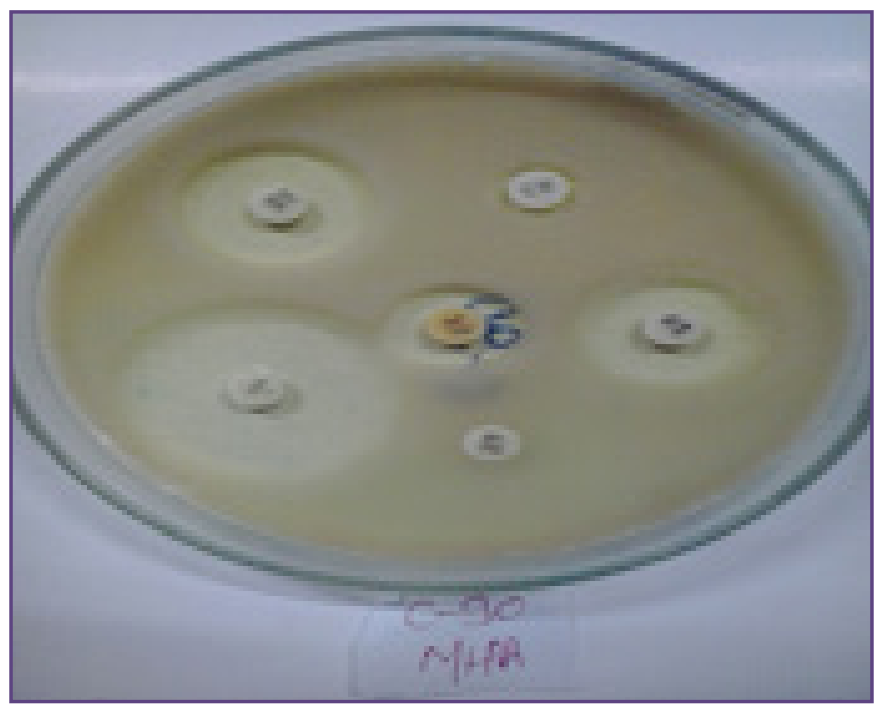

Fig. 1: Antifungal Susceptibility testing of Candida species.

\section{Discussion}

Fungal bloodstream infections are considered to be lifethreatening. They prolong hospital stay and are associated with high morbidity and mortality. ${ }^{[14]}$ Rates of invasive Candida infection have increased significantly, and these infections are a special concern in NICUs. ${ }^{[15]}$ C. albicans is still the Candida species most commonly isolated from neonatal patients with invasive candidiasis, but there has been a significant rise in isolation rates for other nonalbicans Candida species in recent years. ${ }^{[15]}$

In our study the male to female ratio was $1.5: 1$. This is in contrast with the results of caroline et al where female patients were more. ${ }^{[16]}$ In the present study from 118 isolates NAC species accounted for $62.72 \%$ of the cases of neonatal candidemia, whereas C.albicans was responsible for $37.28 \%$ of cases [Table 1]. From NAC 28.81\% (34/118) were c.glabrata, $14.4 \%$ (17/118) were c.parapsilosis, $12.71 \%(15 / 118)$ c.tropicalis were c.parapsilosis and $6.77 \%$ (8/118) were other candida species (c.guilliermondii, $c$. krusei and c. kefyr) [Table 1]. C.albicans was the most common isolated species. This corroborates well with the results of study of Sardana V et al (2012). ${ }^{[17]}$ Prolonged hospitalization, central venous catheterization, previous antibiotic usage, hyperalimentation, surgical procedures, and administration of lipids and steroids are known risk 
factors for Candida infection in both adults and children. [15] In accord with this, all our neonatal patients with candidemia had a history of antibiotic administration.

A recent study has shown that prophylactic fluconazole therapy of very low birth weight (preterm) newborns during their first 6 weeks of life lowers the likelihood of developing serious yeast infections. ${ }^{[18]}$ These results have led to wide adoption of targeted fluconazole prophylaxis strategy in neonatal intensive care units for VLBW infants to prevent invasive candidiasis. ${ }^{[18]}$ If this approach to prophylaxis becomes a standard of care, these NICU infection patterns should be carefully monitored.

Striking feature of the present study was isolation of $C$. glabrata $(28.81 \%)$ and C. parapsilosis $(14.4 \%)$ as the most common NAC species [Table 1]. The NICU was also the hospital location with the highest percentage of C. glabrata isolates that were resistant, although the total number of C. glabrata isolates was less than of C. albicans isolates. Similar results were obtained by other studies also. ${ }^{[19]}$ Although C. parapsilosis is less virulent, but under certain conditions (IV catheters, high IV glucose concentrations) virulence may increase many folds and it is relatively difficult to eradicate this organism. ${ }^{[20]}$ Outbreaks of $C$. parapsilosis BSI in NICUs have been previously reported in the study of Almirante B et al(2006). ${ }^{[21]}$ C. parapsilosis and $C$. glabrata are an emerging fungal pathogen and the major threat for neonates in NICU as it frequently colonizes the hands of $\mathrm{HCW}$, has high affinity for intravascular devices, and parenteral nutrition. ${ }^{[22]} C$. tropicalis is the second leading cause of candidemia in adults, but is quite infrequent among neonates. ${ }^{[23]}$ In our study c.tropicalis was isolated in $12.71 \%$ patients. This might be due to premature and LBW infants have an immature immune system and may behave like an immunocompromised adult patient in this regard. Prophylaxis with azole agents may increase the risk of infection with resistant Candida spp. ${ }^{[24]}$ In a study of 409 ELBW infants compared to historical controls, fluconazole prophylaxis significantly decreased invasive Candida infections and mortality due to Candida infections. ${ }^{[25]}$

Hence determining a pathogen's antifungal susceptibility is an important step in effective treatment. ${ }^{[26]}$ However, it takes considerable time to isolate and identify organisms from patients with invasive fungal infections, and to determine resistance profiles. Delayed initiation of appropriate antifungal therapy can raise the risk of mortality and morbidity in infants, and also increases the likelihood of cross-contamination. ${ }^{[27]}$

In our study from all isolates of candida, fluconazole was resistant in $29.7 \%$, Intermediate in $9.3 \%$ and sensitive in $61.0 \%$ patients [Table 2]. NAC species, especially $C$. glabrata and C. krusei were more resistant to fluconazole than $C$. albicans. This is comparable to SENTRY study in which $C$. albicans and $C$. parapsilosis species were more susceptible to azoles and amphotericin B than C. glabrata. ${ }^{[28]}$. A study by the International Fungal Surveillance Participant Group showed that $90 \%$ of the isolates were susceptible to fluconazole ${ }^{[26]}$ but in our study only $61.1 \%$ patients were susceptible to fluconazole.

Amphotericin B is the drug of choice for treating neonatal candidiasis, and azoles can be good alternatives. ${ }^{[15]}$ In our study Amphotericin B was resistant in 10.1\%, Intermediate in $3.4 \%$ and sensitive in $86.4 \%$ patients[Table 2]. Twelve isolates (5 glabrata, 4 C. albicans, 2 C. tropicalis and 1 c. paropsilosis) were found resistant to amphotericin B. Although resistance to Amphotericin B was quite low $(10.1 \%)$, but is a matter of concern as emergence of such isolates may pose serious therapeutic challenges and also increases risk of nosocomial infection. This is in contrast with other study where amphotericin B was very effective against all Candida isolates and resistance to azoles varied. ${ }^{[29]}$ Dose-dependent susceptibility to fluconazole and amphotericin B was seen in $9.3 \%$ and $3.4 \%$ of the isolates, respectively.

Another study revealed that many Candida bloodstream isolates exhibit decreased susceptibility to systemic antifungal drugs such as amphotericin B and fluconazole. ${ }^{[28]}$

Voriconazole and Itraconazole were 100\% sensitive [Table 2]. Where as in another study from the SCOPE program $90 \%$ of isolates were susceptible to itraconazole. ${ }^{[30]}$ ketoconazole was sensitive in $99.1 \%$ patients[Table 2].The results of this study demonstrate that Voriconazole and Itraconazole were the most active agent in vitro and ketoconazole was next to it with only 1 isolate exhibiting resistance to it. Voriconazole, Itraconazole and ketoconazole were appear to be more active in vitro than fluconazole for several species considered less susceptible to fluconazole namely, C. albicans, C.glabrata, and C. krusei.

\section{Conclusion}

Now a days significance of Candida spp. in NICU is increasing to significant extent. Although previously Candida albicans accounted for majority cases of candidiasis, recently NAC species have been increasingly reported. Among NAC species c.glabrata, c.parapsilosis and c.tropicalis are more commonly isolated species. Although Amphotericin B is the drug of choice for treating neonatal candidiasis, it should be reserved for life threatening conditions. Azoles can be good alternatives for treating these infections. The extended spectrum azole drugs like Voriconazole, Itraconazole and ketoconazole 
may be more effective for treating infections caused by several species of candida which are considered inherently resistant to fluconazole.

\section{Reference}

1. Singhi S, Rao DS, Chakrabarti A. Candida colonization and candidemia in a pediatric intensive care unit. Pediatr Crit Care Med.2008;9:91-5.

2. Stoll BJ, Hansen N, Fanaroff AA, Wright LL, Carlo WA, Ehrenkranz RA, et al. Late-onset sepsis in very low birth weight neonates. The experience of the NICHD Neonatal Research Network. Pediatrics 2002;110:285-91.

3. Ariff S, Saleem AF, Soofi SB, Sajjad R. Clinical spectrum and outcomes of neonatal candidiasis in a tertiary care hospital in Karachi, Pakistan. J Infect Dev Ctries 2011;5:216-23.

4. Beck-sague CM, Jarvis WR. The national nosocomial infections surveillance system secular trends in the epidemiology of nosocomial fungal infections in the United States, 1980-1990 J. Infect Dis.1993;167:1247-51.

5. Nielsen H, Stedemp J, Buin R. Fungemia at a university hospital 1984-1988. Scand J Infect Dis 1991;23:275-82.

6. Benjamin DK Jr, Stoll BJ, Fanaroff AA, McDonald SA, Oh W, Higgins RD et al. National Institute of Child Health and Human Development Neonatal Research Network. Neonatal candidiasis among extremely low birth weight infants: Risk factors, mortality rates, and neuro developmental outcomes at 18 to 22 months. Pediatrics 2006;117:84-92.

7. Elguezabal N, Lopitz-Otsoa F, Lain A, Larrinoa IF, Moragues MD, Ponton J. Serodiagnosis of mycoses using recombinant antigens.Mycopathologia 2005;160:97-109.

8. Prasad KN, Agarwal J, Dixit AK, Tiwari DP, Dhole TN, Ayyagiri A. Role of yeasts as nosocomial pathogens and their susceptibility to fluconazole and amphotericin B. Indian J Med Res 1999;110:11-7.

9. Magill SS, Shields C, Sears CL, Choti M, Merz WG. Triazole cross-resistance among Candida spp.: Case report, occurrence among bloodstream isolates, and implications for antifungal therapy. J Clin Microbiol 2006;44:529-35.

10. Lortholary O, Dupont B. Antifungal prophylaxis during neutropenia and immunodeficiency. Clin Microbiol Rev 1997;10(3):477-04.

11. Hospenthal DR, Murrey CK, Rinaldi MG. The role of antifungal susceptibility testing in the therapy of candidiasis. Diagn. Microbiol.Infect. Dis.2004;48:153-60.

12. National Committee for Clinical Laboratory Standards. Method for antifungal disk diffusion susceptibility for yeasts. Approved guideline M44-A. National Committee for Clinical Laboratory Standards, Wayne, Pa 2004.

13. Chandar J. textbook of medical mycology. 13th ed. New Delhi: Mehta publishers. 2012 p.522-530.

14. Verduynlunel FM, Meis JF, Voss A. Nosocomial fungal infections: candidemia. Diagnostic Microbiology and Infectious Disease 1999;34:213-220.
15. Roilides E, Farmaki E, Evdoridou J, Dotis J, Hatziioannidis E, Tsivitanidou $\mathrm{M}$ et al. Neonatal candidiasis: analysis of epidemiology, drug susceptibility, and molecular typing of causative isolates. European Journal of Clinical Microbiology \& Infectious Diseases2004;23:745-750.

16. Onwuanak CA, Okolo SN, Ige KO, Okpe SE, Toma BO. The effects of birth weight and gender on neonatal mortality in north central Nigeria. BMC Research Notes2011;4:562.

17. Sardana V, Pandey A, Madan M, Goel SP, Asthana AK. Neonatal candidemia: A changing trend. Indian J Pathol Microbiol 2012;55:132-3.

18. Kaufman D, Boyle R, Hazen KC, Patrie JT, Robinson M, Donowitz GL. Fluconazole prophylaxis against fungal colonization and infection in preterm infants. $\mathrm{N}$ Engl $\mathrm{J}$ Med.2001;345:1660-66.

19. Hazen KC, Baron EJ, Colombo AL, Girmenia C, SanchezSousa A, Palacio A et al. Comparison of the Susceptibilities of Candida spp. to Fluconazole and Voriconazole in a 4-Year Global Evaluation Using Disk Diffusion: J C M2003;41(12):5623-32.

20. Capriles CH, Essayag SM, Azpiróz A, Ponente A, Magaldi S, Pérez C, et al. Neonatal candidiasis in Venezuela: Clinical and epidemiological aspects. Rev Latinoam Microbiol 2005;47:11-20.

21. Almirante B, Rodríguez D, Cuenca-Estrella M, Almela M, Sanchez F, Ayats J, et al. Epidemiology, risk factors, and prognosis of Candida parapsilosis bloodstream infections: Case-control population-based surveillance study of patients in Barcelona, Spain, from 2002 to 2003. J Clin Microbiol 2006;44:1681-5.

22. Trofa D, Gácser A, Nosanchuk JD. Candida parapsilosis, an emerging fungal pathogen. Clin Microbiol Rev 2008;21:606-25.

23. Roilides E, Farmaki E, Evdoridou J, Francesconi A, Kasai M, Filioti J, et al. Candida tropicalis in a neonatal intensive care unit: Epidemiologic and molecular analysis of an outbreak of infection with an uncommon neonatal pathogen. J Clin Microbiol 2003;41:735-41.1

24. Zaoutis TE, Foraker E, Mcgowan KL, Mortensen J, Campos $\mathrm{J}$, Walsh TJ et al. Antifungal susceptibility of Candida spp. Isolated from pediatric patients: a survey of 4 children's hospitals. Diagnostic Microbiology and Infectious Disease2005;52:295-98.

25. Healy CM, Campbell JR, Zaccaria E, Baker CJ. Fluconazole prophylaxis in extremely low birth weight neonates reduces invasive candidiasis mortality rates without emergence of fluconazoleresistant Candida species. Pediatrics.2008;121:703-10.

26. Pfaller MA, Diekema DJ, Messer SA, Boyken L, Hollis RJ, Jones RN. In vitro susceptibilities of rare Candida bloodstream isolates to ravuconazole and three comparative antifungal agents. Diagnostic Microbiology and Infectious Disease.2004;48, 101-5. 
27. Avila-Aguero ML, Canas-Coto A, Ulloa-Gutierrez R, Caro MA, Alfaro B, Paris MM. Risk factors for Candida infections in a neonatal intensive care unit in Costa Rica. International Journal of Infectious Diseases. 2005;9:90-5.

28. Pfaller MA, Diekema DJ, Jones RN, Messer SA, Hollis RJ. Trends in antifungal susceptibility of Candida spp. isolated from pediatric and adult patients with bloodstream infections: SENTRY Antimicrobial Surveillance Program, 1997 to 2000. Journal of Clinical Microbiology.2002;40:852-56.
29. Kuzucu C, Durmaz R, Otlu B, Aktas E, Gulcan H, Cizmeci Z. Species distribution, antifungal susceptibility and clonal relatedness of Candida isolates from patients in neonatal and pediatric intensive care units at a medical center in Turkey. New Microbiologica.2008;31:401-8.

30. Pfaller MA, Jones RN, Messer SA, Edmond MB, Wenzel RP. National surveillance of nosocomial bloodstream infection due to Candida albicans: frequency of occurrence and antifungal susceptibility in the SCOPE Program. Diagn Microbiol Infect Dis.1998;31:327-32.

\section{*Corresponding author:}

Dr. Arati Ankushrao Bhadade, 303, Dwaramai apartment, near Dargah, Wanjari Nagar, Nagpur, India

Phone: +91 9975933877

Email: aaratimbbs@gmail.com

Date of Submission : 04.08.2016

Date of Acceptance : 20.12.2016

Financial or other Competing Interests: None.
Date of Publication : 24.02.2017 